\title{
Peripheral blood eosinophilia may be a prognostic biomarker in non-small cell lung cancer patients treated with immunotherapy
}

\author{
Adelaide Alves, Margarida Dias, Sérgio Campainha, Ana Barroso \\ Pulmonology Department, Centro Hospitalar de Vila Nova de Gaia/Espinho, Vila Nova de Gaia, Portugal \\ Contributions: (I) Conception and design: All authors; (II) Administrative support: All authors; (III) Provision of study materials or patients: All \\ authors; (IV) Collection and assembly of data: All authors; (V) Data analysis and interpretation: All authors; (VI) Manuscript writing: All authors; (VII) \\ Final approval of manuscript: All authors. \\ Correspondence to: Adelaide Alves. Pulmonology Department, Centro Hospitalar de Vila Nova de Gaia/Espinho, Rua Conceição Fernandes, $4434-502$ \\ Vila Nova de Gaia, Portugal. Email: adelaide.pereira.alves@gmail.com.
}

\begin{abstract}
Background: Eosinophils have been traditionally associated with the initiation and propagation of inflammatory responses, particularly in allergic diseases and helminth infections. More recently, an association between eosinophils and cancer has been the focus of several studies, but controversial results have emerged. This study aims to evaluate the prognostic role of peripheral blood eosinophilia in nonsmall cell lung cancer (NSCLC) patients receiving immunotherapy (IO). We also evaluated the impact of peripheral eosinophilia on the occurrence of immune-related adverse effects (irAEs).

Methods: Advanced NSCLC patients under IO were included in a retrospective single-center study. Peripheral blood eosinophilia was defined by a count greater than $500 / \mu \mathrm{L}$. Patients were analyzed for eosinophil counts, overall survival (OS), progression-free survival (PFS), overall response rate (ORR) and disease control rate (DCR).

Results: A total of 121 NSCLC patients receiving IO were included. Thirty-three (27.3\%) patients presented peripheral blood eosinophilia during treatment. Patients with peripheral eosinophilia presented more frequently non-progression as best overall response to IO (83.3\% vs. 58.1\%, P=0.014), higher median OS (26.6 vs. 9.5 months, $\mathrm{P}=0.022)$ and higher median PFS (13.8 vs. 4.6 months, $\mathrm{P}=0.013)$. IrAEs were more common in patients with peripheral eosinophilia (66.7\% vs. $36.4 \%, \mathrm{P}=0.003)$.

Conclusions: This study suggests that peripheral blood eosinophilia may predict better outcomes in NSCLC patients receiving IO, despite being associated with an increased risk of irAEs. According to our findings eosinophils may be involved in immune response against tumor. Routine eosinophils count assessment may be an additional prognostic tool in NSCLC patients receiving IO.
\end{abstract}

Keywords: Eosinophil; immune checkpoint inhibitors; advanced non-small-cell lung cancer (advanced NSCLC); prognosis

Submitted Dec 16, 2020. Accepted for publication Mar 05, 2021.

doi: $10.21037 /$ jtd-20-3525

View this article at: http://dx.doi.org/10.21037/jtd-20-3525

\section{Introduction}

Non-small cell lung cancer (NSCLC) accounts for 80-90\% of lung cancers and is the leading cause of cancer death in man and women worldwide (1). Approximately half of all patients diagnosed with NSCLC present metastatic disease at the time of diagnosis, with a 5 -year overall survival (OS) of only about $18 \%$ (2). Immunotherapy (IO) has been used to treat patients with advanced NSCLC through enhancing the immune response to tumor, allowing good clinical outcomes (3). Expression of PD-1 ligand (PD-L1) in tumor cells is the best established biomarker for response to IO but it has been addressed the variability between expression of the PD-L1 and the clinical response to IO in patients with 
advanced NSCLC $(4,5)$. Besides, there is a lack of evidence about which patients will present a durable response to IO and which will have increased risk of immune-related adverse effects (irAEs). All these facts justify the need to identify additional biomarkers of response to IO.

The eosinophil count in peripheral blood seems to be a promising prognostic marker in several cancers $(6,7)$. However, paradoxical data has emerged regarding the role of these cells. In fact, recent studies have shown evidence that eosinophils may have both immunostimulatory and immunoinhibitory functions in tumor growth, according to the type of cancer and different stimuli in the tumor microenvironment (TME) $(8,9)$. A high eosinophil count in peripheral blood has been related with a better prognosis in several cancers $(10,11)$. Nevertheless, an association between eosinophils and a non-beneficial prognosis has also been described $(12,13)$.

The aim of this study was to identify the impact of peripheral blood eosinophil count on prognosis of patients with NSCLC receiving IO. Additionally, we also evaluated if eosinophilia per se is associated with an increased risk of irAEs.

We present the following article in accordance with the STROBE reporting checklist (available at http://dx.doi. org/10.21037/jtd-20-3525).

\section{Methods}

\section{Study design and data collection}

We conducted a retrospective, single-center study of all patients with advanced (stage IIIB to IV) NSCLC treated with IO in monotherapy (in first or subsequent lines) in the Multidisciplinary Department of Thoracic Tumors of Centro Hospitalar de Vila Nova de Gaia/Espinho, in Portugal, between December 2015 and October 2019. Data collection was conducted in December 2019. Exclusion criteria included recent ( $<2$ months) IO treatment initiation which did not allow the evaluation of radiological response at that time and treatment with systemic corticosteroids at the IO initial prescription given the potential to interfere with eosinophil count. Patients were treated with pembrolizumab, nivolumab or atezolizumab. Pembrolizumab was administered intravenously at a dose of $2 \mathrm{mg} / \mathrm{kg}$ every 3 weeks until August 2018 and after that at a dose of $200 \mathrm{mg}$ every 3 weeks; nivolumab was administered intravenously at a dose of $3 \mathrm{mg} / \mathrm{kg}$ every 2 weeks until April 2018 and after that at a dose of $240 \mathrm{mg}$ every 2 weeks; atezolizumab was administered intravenously at a dose of 1,200 mg every 3 weeks. Whenever possible, tumor biopsy specimens were submitted to immunohistochemical staining with monoclonal anti-PD-L1 clone 22C3 (Dako) to quantify PD-L1 expression.

Blood samples (including hemogram with eosinophilic count) were collected from all patients one to 2 days before IO initiation treatment. Thereafter, blood samples were collected on the day before every IO treatment cycle.

Radiological response was evaluated 2 months after IO initiation and thereafter every 3 months. In cases of suspected tumor pseudoprogression, radiological reassessment was performed in 1-2 months.

This study was conducted in accordance with the Declaration of Helsinki (as revised in 2013) and was approved by the institutional Ethics Committee of Centro Hospitalar de Vila Nova de Gaia/Espinho. The requirement for written consent was waived because of the retrospective nature of the study.

\section{Definitions}

There is no consensus in literature regarding the value of peripheral blood eosinophils that may predict response to IO. We defined peripheral blood eosinophilia as a count greater than $500 / \mu \mathrm{L}$, according to a most recent study in this field (6).

Tumor radiological response to IO was evaluated by computed tomography according to the Response Evaluation Criteria in Solid Tumors (RECIST), version 1.1.

Best overall response was defined as progression when there was RECIST criteria of progressive disease and as non-progression when there was RECIST criteria of stable disease, partial response or complete response.

Overall response rate (ORR) was defined as the proportion of patients who had a partial or complete response to therapy. Disease control rate (DCR) was defined as the proportion of patients who had achieved stable disease, partial response or complete response.

Progression-free survival (PFS) was defined as the time from treatment initiation to radiological progression or death from any cause. OS was the time from treatment initiation to death from any cause.

Adverse events were diagnosed and graded according to Common Terminology Criteria for Adverse Events (CTCAE) version 5.0. The grade recorded refers to the highest grade of irAEs that each patient developed. In all 
consultations, patients collected blood tests and were asked about symptoms and possible adverse events.

\section{Statistical analysis}

Descriptive statistics of the variables were expressed as absolute and relative frequencies, mean \pm standard deviation (SD) (if variable with normal distribution) or median and range (if variable without normal distribution). Groups comparisons were carried out by $t$-test or Mann-Whitney test for continuous variables and by the Chi-square test and Fisher test for categorical variables, according to the expected frequencies. The binomial test was used to assess equality of proportions between groups. Survival analysis was estimated using Kaplan-Meier method and differences in OS and PFS were evaluated using the log-rank test. Cox proportional hazard regression model was applied to estimate hazard ratio (HR) and 95\% CI. The level of significance was set to 0.05 . The statistical analysis was conducted using SPSS 22.0 (IBM Corp., Armon, NY, USA).

\section{Results}

\section{Study population}

A total of 131 patients with advanced NSCLC were included in the study. Among them, 10 patients were excluded because they were not evaluable for radiological response assessment. We didn't exclude patients being treated with low dose of systemic corticosteroids at the IO initial prescription ( $\leq 10 \mathrm{mg}$ prednisolone or equivalent). Therefore, 121 patients were included in the analysis. The demographic and clinical characteristics of these patients are shown in Table 1. The most frequent histological type was adenocarcinoma. Analysis of PD-L1 expression in tumor specimens was available in $114(94.2 \%)$ patients and mutational status was known in $86(71.1 \%)$ patients. Among patients who presented peripheral eosinophilia, only one had a past medical history of asthma and no patient had allergic rhinitis or skin disease. Three patients had regular follow-up by a rheumatologist because of rheumatologic disease (Raynaud's disease under investigation, juvenile arthritis and symmetric polyarthritis suspected to be a paraneoplastic disorder).

\section{Blood eosinophil count during treatment}

Twelve patients had pre-treatment peripheral eosinophilia and most of them $(n=10 ; 83.3 \%)$ maintained it during IO treatment. On the other hand, of patients without pretreatment peripheral eosinophilia $(n=109), 23(21.1 \%)$ developed eosinophilia during IO.

Overall, 33 (27.3\%) patients presented eosinophilia in peripheral blood during treatment with IO.

Patients who developed eosinophilia during IO had more frequently rheumatologic diseases (9.1\% vs. $1.1 \%$, $\mathrm{P}=0.029)$, received a higher number of treatment cycles $(14$ vs. $5, \mathrm{P}<0.001)$ and were treated with $\mathrm{IO}$ more months $(10$ vs. $3, \mathrm{P}<0.001)$. No other statistically significant differences between groups were found (Table 1).

\section{Eosinophilia during treatment}

Of patients without previous peripheral blood eosinophilia who developed it during treatment, the median time until eosinophilia was 5.0 months (range, 1.0-7.0) and in most of these patients $(n=17 ; 73.9 \%)$ it occurred in the first 6 months of treatment. One patient developed peripheral blood eosinophilia only 24 months after starting treatment. Median time until peak eosinophils count was 7.0 months (range, 3.0-12.0 months) and the median peak eosinophil count was $770 / \mu \mathrm{L}$ (range, $570-890 / \mu \mathrm{L}$ ). On the other hand, patients with baseline and throughout treatment eosinophilia reached peak eosinophils count earlier and recorded higher eosinophils counts, as shown in Table 2.

The highest peripheral eosinophilia value observed was 61,260 cells $/ \mu \mathrm{L}$, which was found in an asymptomatic patient with baseline peripheral eosinophilia of 4,410 cells $/ \mu \mathrm{L}$. In this patient, treatment with IO was discontinued and due to such an exuberant value of eosinophilia the patient also started treatment with systemic corticosteroids. No evidence of irAEs was found in this patient. No other patient in this study stopped treatment with IO due to peripheral eosinophilia. In fact, the remaining 32 patients with peripheral eosinophilia were asymptomatic, maintained the treatment with IO and were kept under clinical and analytical surveillance. No patient had evidence of helminth infection, but three patients were empirically treated with deworming.

\section{Eosinophilia and treatment outcomes}

Radiological response was not possible to evaluate in 17 patients: 8 patients died before radiological evaluation to treatment, 5 patients experienced irAEs grade 3 and 4 
Table 1 Patient's demographic and clinical characteristics

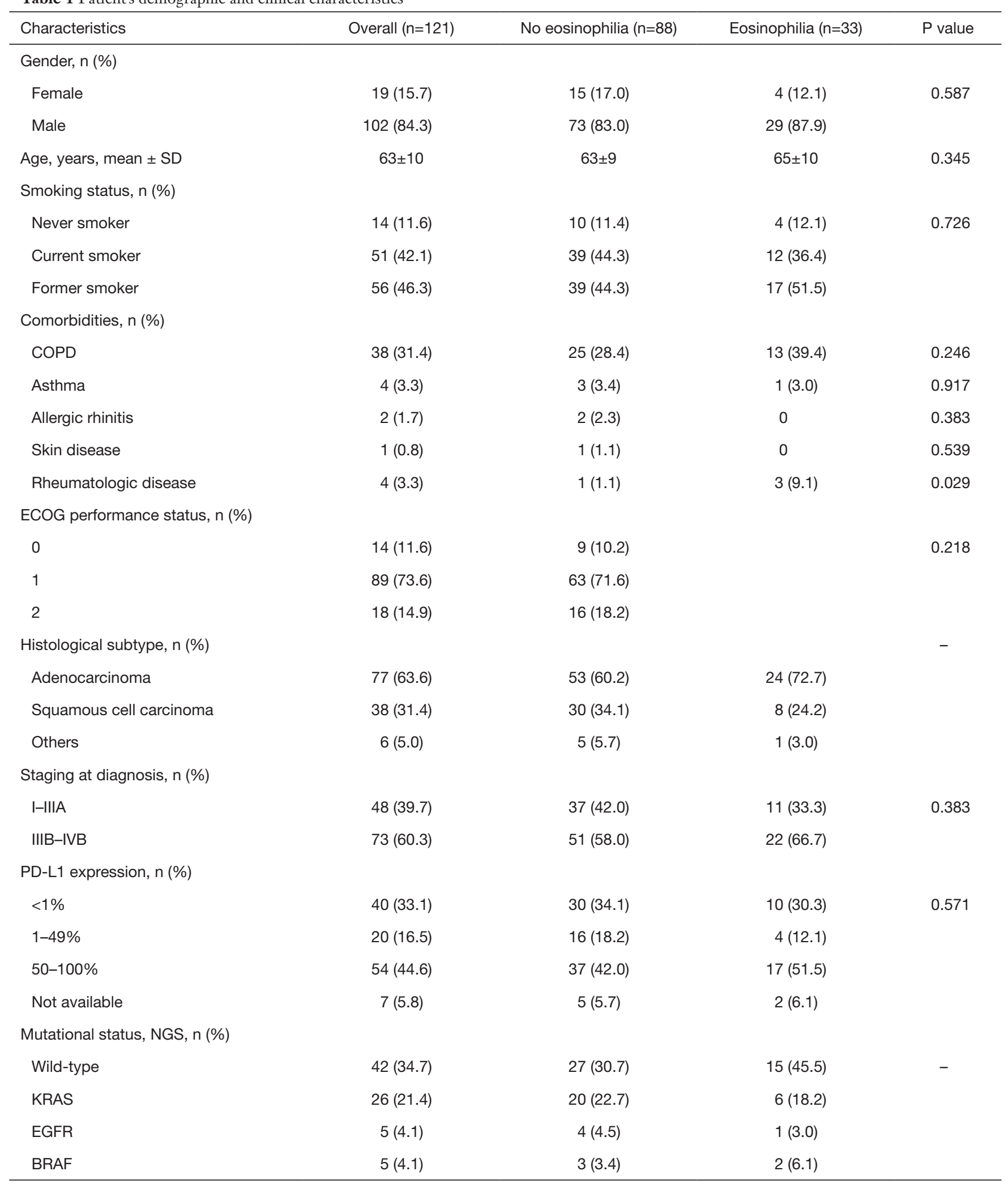

Table 1 (continued) 
Table 1 (continued)

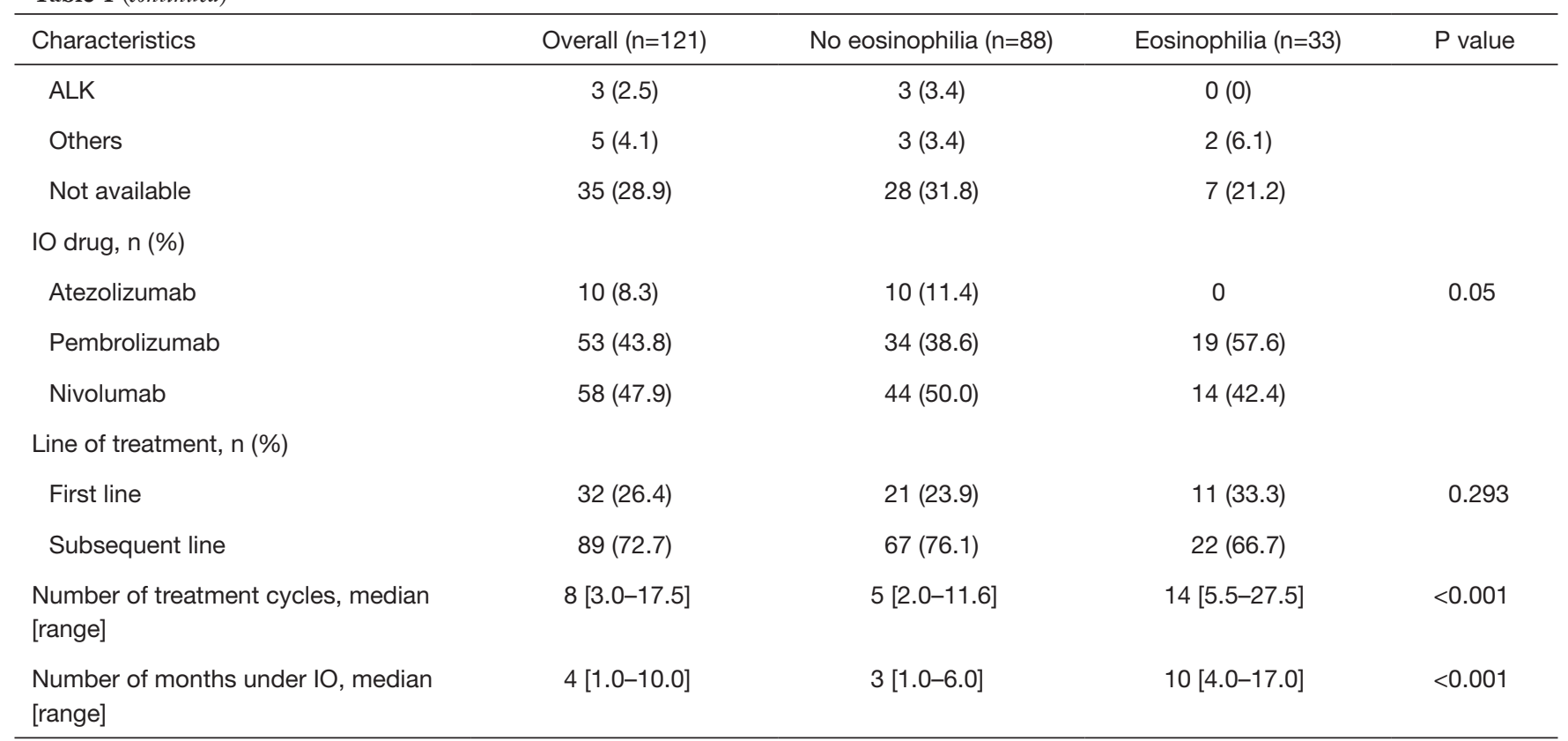

SD, standard deviation; COPD, chronic obstructive pulmonary disease; ECOG, Eastern Cooperative Oncology Group; PD-L1, programmed death 1 ligand; KRAS, Kirsten rat sarcoma viral oncogene homolog; EGFR, epidermal growth factor receptor; BRAF, V-raf murine sarcoma viral oncogene homolog B1; ALK, anaplastic lymphoma kinase; NGS, next generation sequencing; IO, immunotherapy.

Table 2 Patients with peripheral blood eosinophilia during IO treatment

\begin{tabular}{|c|c|c|c|}
\hline & Baseline eosinophilia absent & Baseline eosinophilia present & $P$ value \\
\hline Baseline eosinophil count, median [range] & $240[160-330]$ & $795.0[570-1,860]$ & $<0.001$ \\
\hline Time to eosinophilia, months, median [range] & $5.00[1.0-7.0]$ & - & - \\
\hline Time to peak eosinophils count, months, median [range] & $7.0[3.0-12.0]$ & $1.0[1.0-3.3]$ & 0.002 \\
\hline
\end{tabular}

IO, immunotherapy.

(two cases of pneumonitis, one case of diarrhea, one case of myocarditis and one case of hepatitis) which required IO to be stopped before radiological response assessment, 2 patients needed to be treated with high doses of corticosteroids which led to treatment interruption before radiological response assessment and in 2 patients IO was stopped before radiological assessment because of the worsening clinical status.

Patients with peripheral eosinophilia presented more frequently non-progression as best overall response to IO than those who did not develop peripheral eosinophilia during treatment $(83.3 \%$ vs. $58.1 \%, \mathrm{P}=0.014)$. ORR tended to be higher in patients with peripheral eosinophilia during
IO, but this difference was not statistically significant (40.0\% vs. $23.0 \%, \mathrm{P}=0.08$; Figure 1). Similarly, DCR was higher in patients with peripheral eosinophilia, but again without a statistically significant difference $(83.3 \%$ vs. $64.5 \%$, $\mathrm{P}=0.063$; Figure 1).

At the time of this analysis, in the group of patients with peripheral blood eosinophilia during IO, 5 (15.2\%) had progressive disease and 14 (42.4\%) died. Among patients without peripheral blood eosinophilia, 31 (35.2\%) had progressive disease and $50(56.8 \%)$ died.

Median OS under IO treatment was significantly higher in the group of patients with peripheral eosinophilia during IO (26.6 vs. 9.5 months, $\mathrm{P}=0.022$; Figure 1). The 

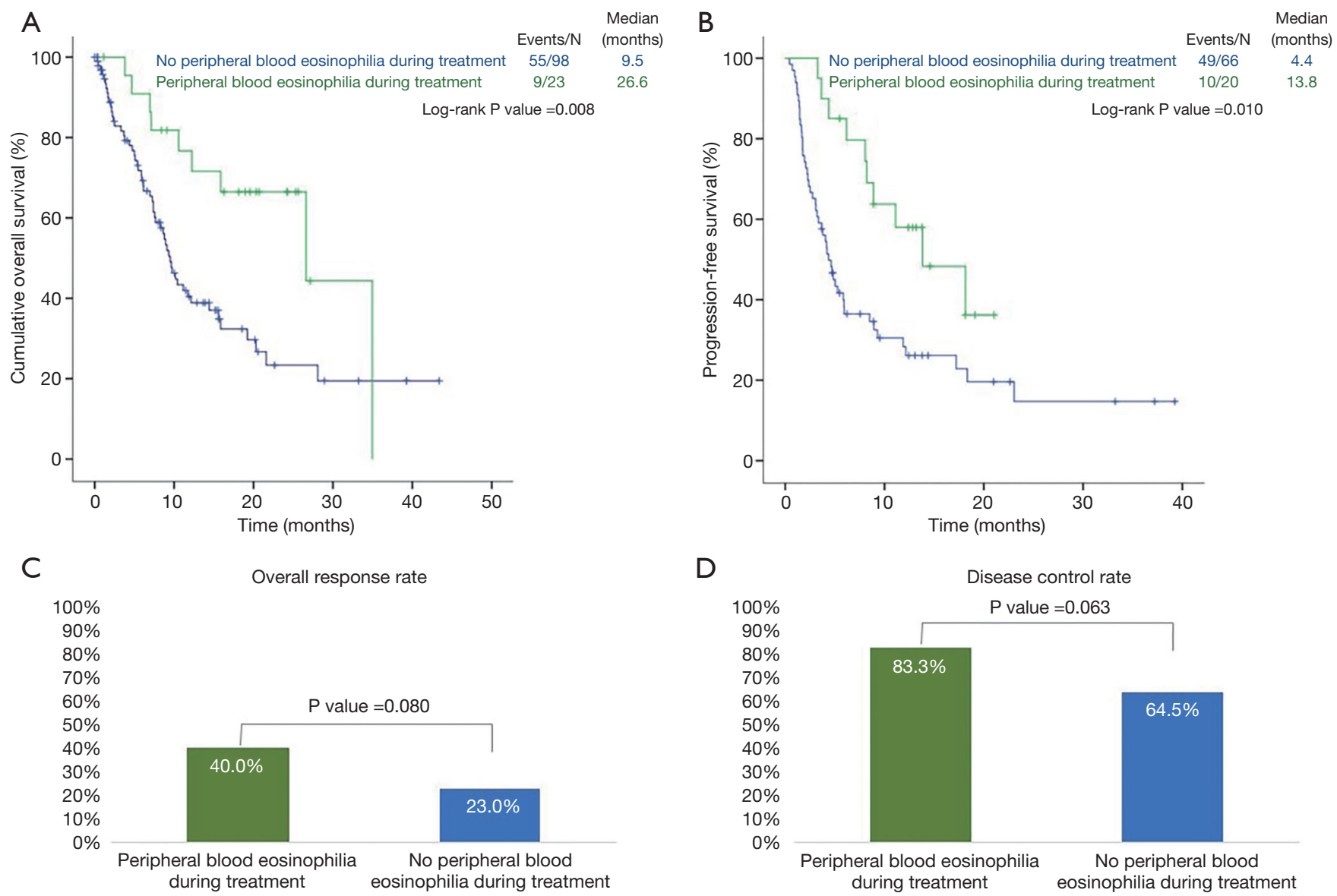

Figure 1 Kaplan-Meier analysis of OS (A) and PFS (B), ORR (C) and DCR (D) for patients with advanced NSCLC receiving IO according to the presence or absence of peripheral blood eosinophilia during treatment. OS, overall survival; PFS, progression-free survival; ORR, overall response rate; DCR, disease control rate; NSCLC, non-small cell lung cancer; IO, immunotherapy.

median PFS was 13.8 months for patients with peripheral eosinophilia and 4.6 months for patient without peripheral eosinophilia ( $\mathrm{P}=0.013$; Figure 1$)$. The presence of peripheral eosinophilia at the beginning of treatment did not interfere with these results.

Multivariate analysis was performed to identify independent prognostic factors related to OS and PFS. Variables with $P$ value $\leq 0.15$ in the univariate analysis were included in the multivariate analysis.

After adjusting for confounding factors, patients with peripheral eosinophilia during treatment tended to have longer OS (HR: $0.779,95 \%$ CI: $0.400-1.518, \mathrm{P}=0.463$ ) and PFS (HR: 0.596, 95\% CI: 0.319-1.111, $\mathrm{P}=0.103$ ), but no statistical difference was found. An Eastern Cooperative Oncology Group (ECOG) 0 or 1 was associated with longer OS (HR: 0.267, 95\% CI: 0.136-0.524, P<0.001) and PFS (HR: 0.402, 95\% CI: 0.211-0.768, P=0.006). The occurrence of irAEs was associated with longer PFS (HR: 0.548, 95\% CI: 0.325-0.923, P=0.024). Smoking habits, PD-L1 expression and number of previous lines of treatment were not independently associated to OS or PFS outcomes. This data suggests that peripheral eosinophilia during treatment may be associated with a favorable clinical response to IO, but others factors may be involved in this association, namely a better performance status and the occurrence of irAEs.

\section{IO, eosinophilia and irAEs}

Patients with peripheral eosinophilia during treatment were more likely to develop irAEs compared to those patients without peripheral eosinophilia (66.7\% vs. $36.4 \%, \mathrm{P}=0.003)$. 
The most frequently documented adverse events were asthenia $(\mathrm{n}=27 ; 22.3 \%)$, hypothyroidism $(\mathrm{n}=8 ; 6.6 \%)$, pneumonitis $(\mathrm{n}=8 ; 6.6 \%)$ and pruritus $(\mathrm{n}=8 ; 6.6 \%)$. Table 3 summarizes the adverse events found in patients under IO. Pruritus was significantly more frequent in patients with peripheral eosinophilia during IO $(\mathrm{P}=0.034)$.

Adverse events led to treatment interruption in 16 (13.2\%) patients: in 6 patients due to pneumonitis (grade 3), in 4 patients due to diarrhea (grades 2 and 3), in 3 patients due to hepatitis (grades 3 and 4), in 2 patients due to myocarditis (grades 3 and 4 ) and in 1 patient due to arthralgia grade 3.

\section{Discussion}

This study reports a high incidence of peripheral blood eosinophilia in patients with NSCLC receiving IO. Although we have chosen a high cut-off value of 500 cells $/ \mu \mathrm{L}$ to define eosinophilia, it was still found in more than a quarter of patients. A previous observational case-series study including 909 patients receiving IO used the same cutoff for peripheral eosinophilia and estimated an incidence of peripheral eosinophilia of $2.8 \%$, which is significantly lower than the incidence of $27.3 \%$ that we reported. Importantly, that study included patients with several advanced cancers and among those who developed eosinophilia $(n=26)$ most had melanoma $(n=20)$ and renal carcinoma $(n=3)$, while our population consisted only of NSCLC patients (6). In fact, our study reports the specific incidence and prognostic impact of peripheral blood eosinophilia in an exclusive population of NSCLC patients receiving IO. We may wonder whether lung cancer itself is particularly associated with an increased risk of peripheral eosinophilia in patients receiving IO compared to other cancers. Lung cancer cells produce IL-5-the most important growth, differentiation and activating factor for eosinophils-which might explain, at least partially, the recruitment of eosinophils into peripheral bloodstream and regional tissue eosinophilia (14-16). High prevalence of peripheral eosinophilia in lung cancer patients may also be explained by smoking. It has been demonstrated that smoking is significantly associated with elevated white blood cell count including eosinophils (17). As it would be expected in lung cancer, most of the patients of our study were current or former smokers, which may also be a reasonable explanation for the high incidence of eosinophilia reported. Among patients with peripheral eosinophilia, only one had a past medical history of allergic disease (asthma). Based on this data, we believe that the development of peripheral eosinophilia during IO may not be associated with a previous background of atopy. Although peripheral eosinophilia was a common finding in this study, none of the patients had symptoms related to it and IO treatment was only discontinued in one patient because of such an exuberant eosinophil count. This data suggests that peripheral blood eosinophilia might be a benign finding in NSCLC patients under IO, seldom requiring other attitudes than surveillance.

The prevalence of ECOG 2 was three times higher among patients who did not develop eosinophilia during treatment than in those who developed it $(18.2 \% \mathrm{vs}$. $6.1 \%, \mathrm{P}>0.05)$. The meaning of this finding is uncertain. Interestingly, these patients with no peripheral eosinophilia tended to be younger and had a lower proportion of advanced disease at diagnosis than those who experienced eosinophilia during treatment. However, patients without peripheral eosinophilia received IO more frequently in subsequent treatment lines than the other group $(76.1 \%$ vs. $66.7 \%, \mathrm{P}>0.05)$. We can speculate that the worse performance status in patients without eosinophilia may be the result of previous lines of treatment, namely with chemotherapy, which may have impaired the ability of the bone marrow to produce white blood cells, including eosinophils.

Other interesting finding is that $9.9 \%$ of patients had peripheral eosinophilia prior to receiving IO. Of those, only $16 \%$ had eosinophil counts normalized during IO. The remaining patients maintained peripheral eosinophilia during treatment, suggesting that patients with baseline increase of eosinophil count are likely to maintain it during IO. Baseline peripheral eosinophilia also seems to be associated with more significant eosinophil counts and earlier time to peak count once treatment is started. We might speculate that patients with higher baseline eosinophil count may already present their own antitumor inflammatory cascade partially triggered and that IO will further result in the additional recruitment of more eosinophils. Nevertheless, inflammation has also been associated with worse outcomes in lung cancer. In fact, increased C-reactive protein and neutrophil-to-lymphocyte ratio in peripheral blood were associated with shorter OS in advanced NSCLC patients. Importantly, these studies did not analyze the prognostic role of peripheral eosinophils (18-20). Most patients of our study developed peripheral eosinophilia only during IO, namely in the first 6 months. Once again, this finding may suggest that IO is somehow associated with eosinophils recruitment and that eosinophils 
Table 3 Adverse events documented during IO

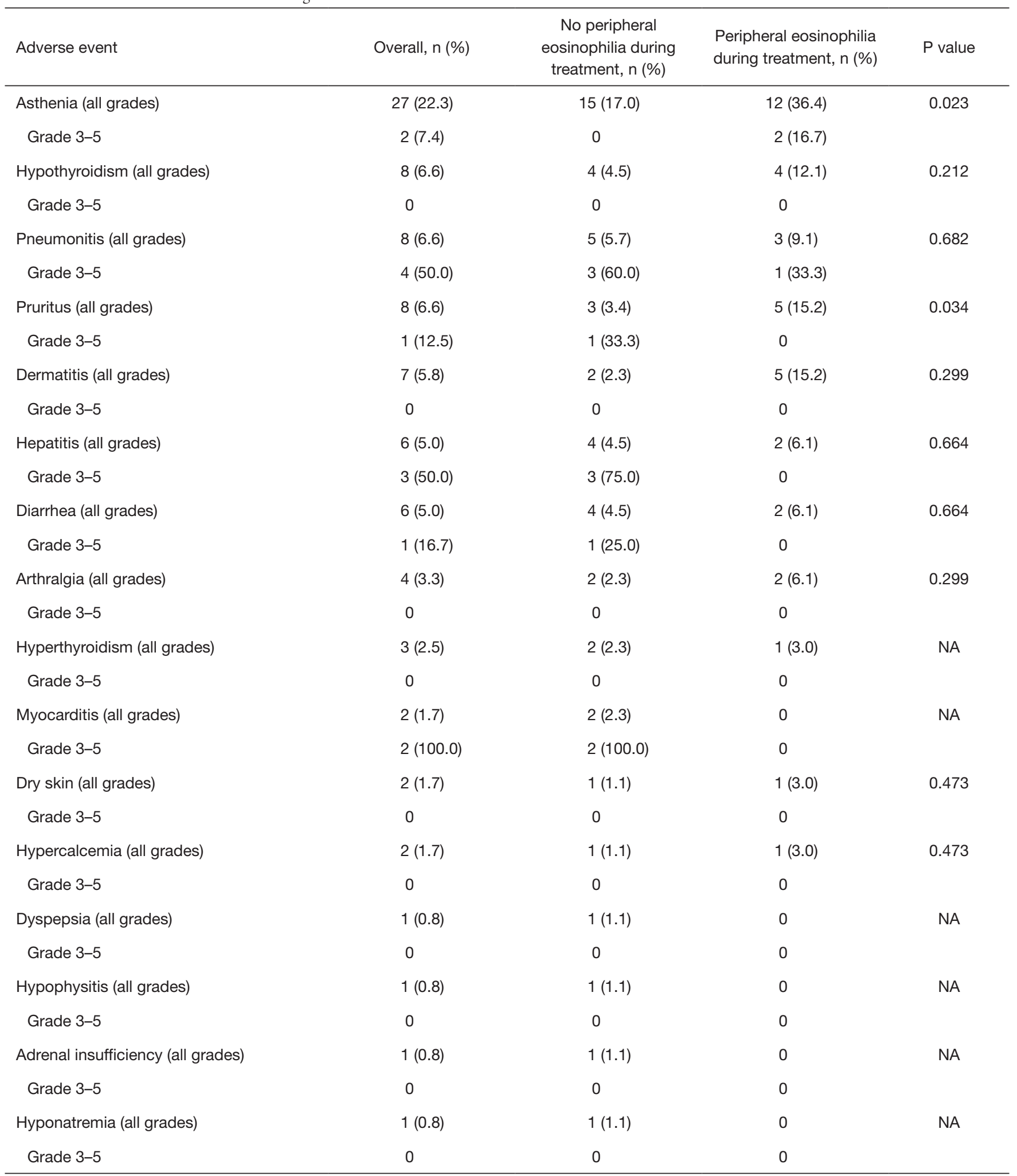

IO, immunotherapy; NA, not available. 
themselves may also be involved in the anti-cancer immune response enhanced by this treatment, representing further evidence regarding the active role of eosinophils in cancer, particularly in NSCLC. At the same time, we may speculate that peripheral eosinophilia could be a marker of IO effectiveness. Human eosinophil itself express PD-L1 but the role of PD-1/PD-L1 axis of eosinophils in tumor biology remains unknown (21).

Our study reports a positive association between peripheral blood eosinophilia during IO treatment and favorable outcomes in NSCLC patients. Accordingly, we found that patients with peripheral eosinophilia were treated with IO over a longer period of time compared to those without peripheral eosinophilia, suggesting that eosinophilia may be potentially associated with more sustained and effective response to IO. Previous studies suggested that eosinophils play antitumor functions as they produce a plethora of chemokines which recruit CD $8^{+}$ $\mathrm{T}$ cells into the TME (22). Depletion of eosinophils was associated with reduced $\mathrm{CD}^{+} \mathrm{T}$ cell infiltration which may weaken the effectiveness of immune response against tumor cells (23). Also, it has been described that eosinophils can exert direct cytotoxic effects against tumor cells by releasing granular content and apoptosis inducing protease granzyme B $(23,24)$. Additionally, angiogenesis, an essential process for tumor growth, may be normalized or suppressed by eosinophils $(22,25)$. IO has yielded good outcomes in cancer patients and peripheral blood eosinophilia has previously been associated with positive response to this treatment $(5,7)$. In a study including metastatic melanoma patients, a positive association between the induction of eosinophilia upon IO and survival has been described and eosinophilia at some point in the course of the disease seemed to be a favorable prognostic marker, even in IO-naive patients (7). In small cell lung cancer, it has been verified a significant inverse correlation between peripheral eosinophil count and metastases, suggesting a protective role of eosinophils in the immune response against this tumor (26). In NSCLC, a high absolute eosinophil count in peripheral blood along with a high absolute lymphocyte count and a low absolute neutrophil count at treatment initiation with nivolumab was significantly associated with a better PFS and OS and may be an independent biomarker (5).

It is important to highlight that other factors may explain the better outcomes found in patients with peripheral eosinophilia during treatment. Patients with peripheral eosinophilia tended to have better ECOG performance status, with a higher proportion of patients with ECOG 0 and 1 compared to patients without peripheral eosinophilia. In fact, when multivariate analysis was conducted, a better ECOG was associated with longer OS and PFS and therefore the beneficial effect of peripheral eosinophilia during IO may be the result of a better general health status found in these patients. Although patients with peripheral eosinophilia were more frequently never smokers, had a higher prevalence of PD-L1 expression between 50-100\% and had received IO as first line treatment more frequently, these potential confounders were not independently associated to a better OS or PFS.

It is not clear whether the predictive role of eosinophilia also applies to patients treated with chemotherapy. In a retrospective study including patients with stage I-III breast cancer, 552 patients out of 930 received neoadjuvant and/ or adjuvant chemotherapy. The authors observed that a higher relative eosinophil count (using a cut-off of $1.5 \%$ ) at baseline was associated to a better prognosis, especially in patients not treated with chemotherapy or with anti-HER2 drugs (27). These results are in line with a previous study in advanced melanoma, according to which a baseline relative eosinophil count $\geq 1.5 \%$ reduced the risk of mortality in patients receiving IO, but had no prognostic value for patients treated with chemotherapy (28). The authors hypothesized that chemotherapy effects on bone marrow leads to a lower production and differentiation of white blood cells, reducing the number of circulating eosinophils during anticancer treatment.

Interestingly, in our study peripheral blood eosinophilia was associated with an increased risk of irAEs. We might speculate that if eosinophils may contribute to boost inflammatory response against tumor cells, these cells may at the same time be involved in the constellation of inflammatory toxicities associated with immune checkpoint inhibitors. Nevertheless, most irAEs were mild and in most cases there was no need of IO cessation. Of note, although irAEs were more common in patients with peripheral eosinophilia, these patients still had better outcomes. In fact, we found that irAEs themselves were associated with a better PFS. Previous studies reported that irAEs may be predictive of IO response in several cancers, including NSCLC. In most of these studies, the occurrence of irAEs was also associated with improvements in PFS, OS and ORR (29-31). More recently, it has been documented that a baseline feature of high peripheral blood eosinophil count $\left(\geq 0.125 \times 10^{9}\right.$ cells/L) was associated with an increased risk of immune-related pneumonitis and with an improvement in PFS (32). It is possible that the increased risk of irAEs we 
found in the group of patients with peripheral eosinophilia may be explained by the longer duration of IO treatment described in these patients, which would mean a longer exposure time. On the other hand, it is also questionable whether irAEs are the cause or the consequence of peripheral eosinophilia. More studies are needed to further clarify the relationship between eosinophilia and irAEs.

This study documents a link between eosinophils, response to IO and irAEs in NSCLC patients, reporting that peripheral eosinophilia may be associated with better and more sustained response to $\mathrm{IO}$ even though an increased risk of irAEs. However, there are some limitations to the present study. First, this is a retrospective observational study lacking a control group of patients who did not receive IO. Second, there is no consensus in literature regarding the ideal cut-off for peripheral eosinophilia, so the definition of more than 500 eosinophils $/ \mu \mathrm{L}$ may not be the best peripheral eosinophils predictor cut-off value. Third, a larger sample size could potentially allow to draw more definitive conclusions.

In conclusion, this study reports a positive association between peripheral blood eosinophilia during IO and improved response to this treatment, although other factors may be involved in this association, namely the general health status of the patients and the occurrence of irAES. Further studies including a larger number of patients are needed to better understand the role of eosinophils in NSCLC patients treated with IO. Our data suggests that peripheral blood eosinophils count may be an additional, easily measured and accessible prognostic biomarker in NSCLC patients under IO. In addition to its prognostic role, eosinophils could also potentially be the specific target of future NSCLC therapies.

\section{Acknowledgments}

The authors are grateful for the statistical support provided by João Cachulo.

Funding: None.

\section{Footnote}

Reporting Checklist: The authors have completed the STROBE reporting checklist. Available at http://dx.doi. org/10.21037/jtd-20-3525

Data Sharing Statement: Available at http://dx.doi. org/10.21037/jtd-20-3525
Conflicts of Interest: All authors have completed the ICMJE uniform disclosure form (available at http://dx.doi. org/10.21037/jtd-20-3525). AB reports personal fees from AstraZeneca, personal fees from BMS, personal fees from Boehringer-Ingelheim, personal fees from Eli Lilly, personal fees from MSD, personal fees from Novartis, personal fees from Pfizer, personal fees from Roche, outside the submitted work. The other authors have no conflicts of interest to declare.

Ethical Statement: The authors are accountable for all aspects of the work in ensuring that questions related to the accuracy or integrity of any part of the work are appropriately investigated and resolved. This study was conducted in accordance with the Declaration of Helsinki (as revised in 2013) and was approved by the institutional Ethics Committee of Centro Hospitalar de Vila Nova de Gaia/Espinho. The requirement for written consent was waived because of the retrospective nature of the study.

Open Access Statement: This is an Open Access article distributed in accordance with the Creative Commons Attribution-NonCommercial-NoDerivs 4.0 International License (CC BY-NC-ND 4.0), which permits the noncommercial replication and distribution of the article with the strict proviso that no changes or edits are made and the original work is properly cited (including links to both the formal publication through the relevant DOI and the license). See: https://creativecommons.org/licenses/by-nc-nd/4.0/.

\section{References}

1. Bray F, Ferlay J, Soerjomataram I, et al. Global cancer statistics 2018: GLOBOCAN estimates of incidence and mortality worldwide for 36 cancers in 185 countries. CA Cancer J Clin 2018;68:394-424.

2. Siegel RL, Miller KD, Jemal A. Cancer statistics, 2016. CA Cancer J Clin 2016;66:7-30.

3. Carter BW, Halpenny DF, Ginsberg MS, et al. Immunotherapy in non-small cell lung cancer treatment: current status and the role of imaging. J Thorac Imaging 2017;32:300-12.

4. Patel SP, Kurzrock R. PD-L1 expression as a predictive biomarker in cancer immunotherapy. Mol Cancer Ther 2015;14:847-56.

5. Tanizaki J, Haratani K, Hayashi H, et al. Peripheral blood biomarkers associated with clinical outcome in non-small cell lung cancer patients treated with nivolumab. J Thorac 
Oncol 2018;13:97-105.

6. Bernard-Tessier A, Jeanville P, Champiat S, et al. Immunerelated eosinophilia induced by anti-programmed death 1 or death-ligand 1 antibodies. Eur J Cancer 2017;81:135-7.

7. Moreira A, Leisgang W, Schuler G, et al. Eosinophilic count as a biomarker for prognosis of melanoma patients and its importance in the response to immunotherapy. Immunotherapy 2017;9:115-21.

8. Simon SCS, Utikal J, Umansky V. Opposing roles of eosinophils in cancer. Cancer Immunol Immunother 2019;68:823-33.

9. Wong DTW, Bowen SM, Elovic A, et al. Eosinophil ablation and tumor development. Oral Oncol 1999;35:496-501.

10. Prizment AE, Anderson KE, Visvanathan K, et al. Inverse association of eosinophil count with colorectal cancer incidence: Atherosclerosis Risk in Communities study. Cancer Epidemiol Biomarkers Prev 2011;20:1861-4.

11. Ownby HE, Roi LD, Isenberg RR, et al. Peripheral lymphocyte and eosinophil counts as indicators of prognosis in primary breast cancer. Cancer 1983;52:126-30.

12. Xie F, Liu LB, Shang WQ, et al. The infiltration and functional regulation of eosinophils induced by TSLP promote the proliferation of cervical cancer cell. Cancer Lett 2015;364:106-17.

13. von Wasielewski R, Seth S, Franklin J, et al. Tissue eosinophilia correlates strongly with poor prognosis in nodular sclerosing Hodgkin's disease, allowing for known prognostic factors. Blood 2000;95:1207-13.

14. Huang M, Wang J, Lee P, et al. Human non-small cell lung cancer cells express a type 2 cytokine pattern. Cancer Res 1995;55:3847-53.

15. Pandit R, Aaron S, Wulfekuhler L, et al. Non-small-cell lung cancer associated with excessive eosinophilia and secretion of interleukin-5 as a paraneoplastic syndrome. Am J Hematol 2007;82:234-7.

16. Slungaard A, Ascensao J, Zanjani E, et al. Pulmonary carcinoma with eosinophilia. Demonstration of a tumor-derived eosinophilopoietic factor. N Engl J Med 1983;309:778-81.

17. Higuchi T, Omata F, Tsuchihashi K, et al. Current cigarette smoking is a reversible cause of elevated white blood cell count: Cross-sectional and longitudinal studies. Prev Med Rep 2016;4:417-22.

18. Machado D, Marques C, Dias M, et al. Inflammatory prognostic biomarkers in advanced non-small cell lung cancer. Pulmonology 2019;25:181-3.

19. Shrotriya S, Walsh D, Bennani-Baiti N, et al. C-reactive protein is an important biomarker for prognosis tumor recurrence and treatment response in adult solid tumors: a systematic review. PLoS One 2015;10:e0143080.

20. Dirican N, Dirican A, Anar C, et al. A new inflammatory prognostic index, based on $\mathrm{C}$-reactive protein, the neutrophil to lymphocyte ratio and serum albumin is useful for predicting prognosis in non-small cell lung cancer cases. Asian Pac J Cancer Prev 2016;17:5101-6.

21. Pesce $\mathrm{S}$, Thoren FB, Cantoni C, et al. The innate immune cross talk between NK cells and eosinophils is regulated by the interaction of natural cytotoxicity receptors with eosinophil surface ligands. Front Immunol 2017;8:510.

22. Carretero R, Sektioglu IM, Garbi N, et al. Eosinophils orchestrate cancer rejection by normalizing tumor vessels and enhancing infiltration of CD $8+T$ cells. Nat Immunol 2015;16:609-17.

23. Lucarini V, Ziccheddu G, Macchia I, et al. IL-33 restricts tumor growth and inhibits pulmonary metastasis in melanoma-bearing mice through eosinophils. Oncoimmunology 2017;6:e1317420.

24. Costain DJ, Guha AK, Liwski RS, et al. Murine hypodense eosinophils induce tumour cell apoptosis by a granzyme B-dependent mechanism. Cancer Immunol Immunother 2001;50:293-9.

25. Xing Y, Tian Y, Kurosawa T, et al. CCL11-induced eosinophils inhibit the formation of blood vessels and cause tumor necrosis. Genes Cells 2016;21:624-38.

26. Shelton A, Green RH, Bradding P, et al. Peripheral blood eosinophil count correlates with survival in lung cancer. Lung Cancer 2010;67:S40-1.

27. Onesti CE, Josse C, Boulet D, et al. Blood eosinophilic relative count is prognostic for breast cancer and associated with the presence of tumor at diagnosis and at time of relapse. Oncoimmunology 2020;9:1761176.

28. Ferrucci PF, Gandini S, Cocorocchio E, et al. Baseline relative eosinophil count as a predictive biomarker for ipilimumab treatment in advanced melanoma. Oncotarget 2017;8:79809-15.

29. Das S, Johnson DB. Immune-related adverse events and anti-tumor efficacy of immune checkpoint inhibitors. J Immunother Cancer 2019;7:306.

30. Toi Y, Sugawara S, Kawashima Y, et al. Association of immune-related adverse events with clinical benefit in patients with advanced non-small-cell lung cancer treated with nivolumab. Oncologist 2018;23:1358-65.

31. Grangeon M, Tomasini P, Chaleat S, et al. Association between immune-related adverse events and efficacy of immune checkpoint inhibitors in non-small-cell lung 
cancer. Clin Lung Cancer 2019;20:201-7.

32. Chu X, Zhao J, Zhou J, et al. Association of baseline peripheral-blood eosinophil count with immune checkpoint inhibitor-related pneumonitis and clinical outcomes in patients with non-small cell lung cancer receiving immune checkpoint inhibitors. Lung Cancer 2020;150:76-82.

Cite this article as: Alves A, Dias M, Campainha S, Barroso A. Peripheral blood eosinophilia may be a prognostic biomarker in non-small cell lung cancer patients treated with immunotherapy. J Thorac Dis 2021;13(5):2716-2727. doi: 10.21037/jtd-20-3525 